attempted for all patients if necessary. For the waist pre-releasing method, the waist of AOD was released immediately following the expansion of the distal umbrella and withdrawn to wedge the defect in order to enhance the self-centralisation of the occluder; for the dumbbell-shaped releasing method, the distal umbrella was released within the upper left pulmonary vein to constrain the expansion of the umbrella, and the delivering catheter was further withdrawn slowly until the proximal umbrella was expanded in the right atrium, and finally, the original shape of the distal umbrella could quickly recover by slightly shaking or pulling the catheter, meanwhile the AOD could well fixed the defect.

Results The average diameter of ASDs in group A and B were $27.2 \pm 11.7 \mathrm{~mm}$ and $28.5 \pm 11.9 \mathrm{~mm}$, respectively ( $p>0.05$ ), and the average diameter of finally used AODs was $34.5 \pm 10.2 \mathrm{~mm}$ and $38.7 \pm 11.9 \mathrm{~mm}$, respectively $(\mathrm{p}<0.01)$. The technical successful rates respectively for group $A$ and $B$ were $61.5 \%$ and $26.1 \%(p<0.05)$ by using the conventional releasing method, increased to $70.1 \%$ and $39.1 \%(p<0.05)$ by trying the waist pre-releasing method, and further increased to $100 \%$ and $100 \%$ by attempting the dumbbellshaped releasing method. Neither major complications no occluder dislodging occurred peri-procedurally in the two groups.

Conclusions Trans-catheter closure of no rim large-to-huge ASD with the AOD may be safe and feasible; closuring no rim large-tohuge ASD needs bigger AODs and more use of the dumbbell-shaped releasing method.

\section{Q0487 ONE YEAR OUTCOME OF TRANSCATHETER CLOSURE OF VERY LARGE ATRIAL SEPTAL DEFECT WITH AMPLATZER OCCLUDERS}

doi:10.1136/hrt.2010.208967.487

Chen Lianglong, Luo Yukun, Lin Chaogui, Peng Yafei, Zheng Xingchun. Union Hospital Fujian Medical University

Objective This study was to investigate the outcome of transcatheter closuring large-to-huge ASD with the AOD within one year.

Methods 35 consecutive patients with large-to-huge ASD (330 mm) underwent transcatheter defect closure and then were followed up peri-procedurally, at 1-, 6-, and 12-month periods following the operation by clinical assessment, electrocardiographic and echocardiographic examination. All patients received 6-month antithromboembolic therapy by using either aspirin or warfarin at the discretion of the operator. The major adverse cardiac events (MACE) include cardiac death, occluder dislodgment leading to urgent cardiac surgery, occluder occupation significantly impeding haemodynamics or cardio-electrical activities, AOD-related thromboembolism, AOD-related atrial rupture, the minor adverse cardiac events (MACE) include occluder occupation with or without slight interference of haemodynamics, AOD-related arrhythmia and residual shunting.

Results The average diameter of ASD in 35 patients was $33.7 \pm 5.2 \mathrm{~mm}$ (range $30 \mathrm{~mm}$ to $38 \mathrm{~mm}$ ), and the average diameter of final AOD used was $38.1 \pm 7.1 \mathrm{~mm}$ (range $32 \mathrm{~mm}$ to $42 \mathrm{~mm}$ ). The immediate technical success was $100 \%$ without severe peri-procedural complications. MACE was not found in each stage within a one year follow-up, but MAC Ewas frequently encountered, among which occluder occupation with asympathetic haemodynamic interference occurred $45.7 \%$ peri-procedurally, $42.9 \%$ at 1 -month, $40.0 \%$ at 6 -month, and $34.3 \%$ at 12 -month, and AOD-related atrial arrhythmia occurred $51.4 \%$ peri-procedurally, $14.3 \%$ at 1 -month, $8.6 \%$ at 6 -month, and $2.9 \%$ at 12 -month. Persistent small residual shunting was found in $24(68.6 \%)$ patients and I ${ }^{\circ}$ AVB in one $(2.9 \%)$ patient.
Conclusions The large-to-huge ASD can be occluded by using AOD without technical difficulty, but the long-term safety and efficacy requires further study.

\section{e0488 TRANSCATHETER CLOSURE OF PARAPROSTHETIC VALVE LEAKS AFTER SURGICAL VALVE REPLACEMENTS}

doi:10.1136/hrt.2010.208967.488

Pan Xin, Zhang Wei, Wu Weihua, Lu Jing, Wang Cheng, Feng Yun, Fang Weiyi. Department of Cardiology, Shanghai Chest Hospital, Shanghai Jiaotong University, Shanghai 200030, China

Objective To elucidate the techniques of transcatheter closure of paravalvular leak (PVL) by Amplatzer occluder, and evaluate its feasibility, effectiveness, and safety.

Methods 5 patients with PVL ( 4 males and 1 female), with a mean age of $58.6 \pm 17.2$ (ranged from 38 to 81 ). Aortic mechanical valve (2), mitral bioprosthetic valve (2), and double mechanical valves (1) replacements were involved. We attempted percutaneous transcatheter closure of PVLs in 4 patients of single valve replacements. Patients requiring double mechanical valves replacement were treated by a team formed by interventionalist and cardiac surgeons. The team used a mini thoracotomy with direct transapical puncture of the left ventricle via a 'hybrid' approach, in order to close mitral PVL under general anaesthesia.

Results We used Chinese-made Amplatzer occluder to close PVLs. All procedures were technically successful. 5 patients of our group reported significant symptomatic improvements during short term follow-up after procedure. 2 patients of aortic PVLs had achieved complete occlusion without residual regurgitation, 3 patients of mitral PVLs closure demonstrated that there had been tiny or mild mitral paraprosthetic leak. However, no interference with valve leaflet motion were diagnosed by transthoracic echocardiography after the procedure. In our group, cardiac perforation and pericardium tamponade occurred in 1 patient with aortic PVL during interventional therapy, who recovered after being given emergent pericardiocentesis. Other 3 patients treated by mitral PVLs closure had transient severe haemolysis, which were resolved after 1 to 3 weeks.

Conclusions Percutaneous or transapical left ventricular access closure of PVLs is technically demanding, but is feasible and safe in selected patients, with a reasonable degree of technical and clinical success. However, there are still a variety of complications, technical and device improvement. Methodology standardisation is further required.

\section{e0489 PULMONARY VEIN STENTING FOR THE TREATMENT OF ACQUIRED SEVERE PULMONARY VEIN STENOSIS COMPLICATING ABLATION FOR ATRIAL FIBRILLATION}

doi:10.1136/hrt.2010.208967.489

Pan Xin, Fang Weiyi, Wu Weihua, Wang Cheng, Lu Jing. Department of Cardiology, Shanghai Chest Hospital, Shanghai Jiaotong University, Shanghai, China

Introduction Ablation procedures for atrial fibrillation (AF) are being performed with increasing frequency. One of the most serious complications is the development of pulmonary vein stenosis, which occurs in 1\% to 3\% of current series. The preferred therapy for symptomatic PVS is pulmonary vein (PV) angioplasty, but this treatment modality is still uncertain in China. The aim of this study 
was the evaluation of the short-term success and safety after PV stenting of severe stenosis.

Methods and results 5 patients (pts) with 11 PVS were prospectively evaluated. All 5 pts experienced onset of dyspnoea on exertion some weeks or several months before, 3 with dyspnoea at rest, 5 with cough, and 2 with chest pain. On multirow spiral CT examination for each case, the narrowest lumen of the affected PVs measured $2 \pm 1.1 \mathrm{~mm}$ compared with $10 \pm 1.3 \mathrm{~mm}$ at baseline $(p \leqq 0.001)$. The length of narrow segment was $10 \pm 2.9 \mathrm{~mm}$. All of 11 veins were treated with stent implantation with Palmaz Genesis stents, which size were 7- or 8-mm-diameter and 12 -, 15-, 18 -mm-length. At percutaneous intervention, these veins showed $81 \pm 13 \%$ stenosis, with a mean gradient of $20 \pm 5 \mathrm{~mm} \mathrm{Hg}$. This was significantly reduced to a residual stenosis of $8 \pm 2 \%(p \leq 0.001)$ and a residual gradient of $3 \pm 2 \mathrm{~mm} \mathrm{Hg} \quad(p \leq 0.001)$ immediately. The mean pulmonary pressure was $46 \pm 5 \mathrm{~mm} \mathrm{Hg}$ pre-intervention, which decreased to $28 \pm 4 \mathrm{~mm} \mathrm{Hg}$ post-intervention in our protocols. The procedure was safety and successful, no complications were occurred in our pts. The symptomatic response was improved immediately and significantly.

Conclusions Percutaneous intervention produces rapid and dramatic symptom relief in patients with highly symptomatic PV stenosis after radiofrequency ablation for AF. Nevertheless, mid-term and long-term follow-up study with flow determination in the affected vein is still need to assess.

\section{E0490 SAFETY AND FEASIBILITY OF TRANSRADIAL CORONARY ANGIOGRAPHY AT THE OUTPATIENT CLINIC}

doi:10.1136/hrt.2010.208967.490

Yang Shiwei, Zhou Yujie, Hu Dayi, Shi Dongmei, Yang Qing, Wang Jianlong, Cheng Wanjun, Nie Bin, Wang Zhijian, Li Yunzhi, Liu Xiaoli. Beijing Anzhen Hospital Affiliated to Capital Medical University

Objective To evaluate the safety and feasibility of transradial coronary angiography at the outpatient clinic.

Methods From February, 2008 to June, 2008, 100 outpatients who received transradial coronary angiography in Anzhen Hospital were included in this analysis, and 100 inpatients who underwent coronary angiography were selected as control group. Primary endpoints included success rate, percentage of angiographic catheter use with different diameters, adverse events during the procedure (such as death, malignant arrhythmia, acute myocardial infarction, coronary artery spasm, coronary artery dissection, perforation or occlusion, etc.) and after the procedure (such as death, acute myocardial infarction, upper limb haematoma, osteofascial compartment syndrome, radial artery pseudoaneurysm or occlusion, etc.).

Results The success rate $(100 \%$ vs $100 \%)$, procedure duration time $(12.5 \pm 3.4) \mathrm{min}$ vs $(10.8 \pm 3.6) \mathrm{min}, \mathrm{p}=0.517)$ and exposition time $(4.3 \pm 1.0) \mathrm{min}$ vs $(4.1 \pm 1.0) \mathrm{min}, \mathrm{p}=0.629)$ were similar between the outpatient and inpatient groups. Radial and coronary artery spasm were the main adverse events during the angiography, and haematoma was the main adverse event after the angiography. There were no significant differences of adverse events between the 2 groups. the total cost of the outpatient group was significantly lower than the inpatient control group $(4012 \pm 238)$ yuan vs $(5329 \pm 371)$ yuan, $\mathrm{p}<0.01$ ). Expenditure including chemical tests, medicine, nursing care, Room \& Board all decreased significantly.

Conclusion Transradial coronary angiography application at the outpatient clinic was safe and feasible for stable patients, and this procedure could decrease the medical expenditure and shorten the admission time.

\section{E0491 ASSOCIATION OF CORONARY HEART DISEASE WITH CAROTID ARTERY INTIMA-MEDIA THICKNESS AND BAPWV}

doi:10.1136/hrt.2010.208967.491

Liu Junming, Huang Menjun, Wang Mingjian, Xie Wei, Dou Qinghui, Zou Qi. Department of Cardiovascular Internal Medicine, Bingtuan Hospital of Production and Construction, China

Objective To investigate the association of coronary heart disease with carotid artery intima-media thickness and baPWV, by measuring carotid intimia - media thickness and baPWV.

Methods 160 patients who had been examined by coronary angiography were divided into normal group and coronary artery disease group (this group was subdivided into 1 vessel group and multi-vessel group). Carotid ultrasonography and baPWV were performed on them

Results Compared to the patients in normal group, The carotid artery IMT of the coronary artery disease group increased significantly with the aggravation of coronary artery stenesis (the norma group: $0.83 \pm 0.08 \mathrm{~mm}$, the 1 vessel group: $0.91 \pm 0.11 \mathrm{~mm}$, the multivessel group: $1.08 \pm 0.15 \mathrm{~mm}, \mathrm{p}<0.01$ ). The baPWV increased too (the normal group $1411.20 \pm 197.71$, the 1 vessel group $1742 \pm 200.89$, the multi-vessel group $2839.40 \pm 319.03, \mathrm{p}<0.01)$. The carotid artery IMT and baPWV were significantly higher in multi-vessel group than those in 1 vessel group and normal group $(p<0.01)$.

Conclusion Carotid atherosclerosis and baPWV have great value in predicting coronary artery disease. For the coronary heart disease high risk group, routine carotid ultrasound examine and the measure of baPWV can be used to estimate the state of coronary artery atherosclerosis.

\section{e0492 CAROTID INTIMIA-MEDIA THICKNESS AND CORONARY ARTERY DISEASE}

doi:10.1136/hrt.2010.208967.492

Liu Junming, Huang Wenjun, Wang Mingjian, Xie Wei, Dou Qinghui, Zou Oi. Department of Cardiovascular Internal Medicine, Bingtuan Hospital of Production and Construction, Xinjiang, China

Objective To analyse the relationship of carotid atherosclerotic and coronary artery disease (CAD), by measuring Carotid intimia media thickness.

Methods The plaque index and intimia - media thickness (IMT) were detected by high-resolution ultrasound in patients with coronary artery angiography. The extent of difference of IMT were compared between patients with and without coronary artery disease.

Results Compared to the patients with no CAD, the IMT of patients with CAD significantly increased $(0.83 \pm 0.08 \mathrm{~mm}$ vs $0.91 \pm 0.11 \mathrm{~mm} ; 0.83 \pm 0.08 \mathrm{~mm}$ MS1.08 $\pm 0.15 \mathrm{~mm} ; 0.91 \pm 0.11 \mathrm{~mm}$ MS1.08 $\pm 0.15 \mathrm{~mm} ; \mathrm{p}<0.05)$. The incidence of plaque in CAD patients was higher than that of patients with no CAD. The IMT was significantly higher in multi-vessel group than those in univessel group $\mathrm{p}<0.05)$.

Conclusion In CAD patients, the widened inner diameter and lower blood flow rate of carotid artery result in a maximal shearing force significantly lower than that of the control group.The increasing intima-media thickness is associated greatly with carotid atherosclerosis. The haemodynamics change plays an important role in the development and progression of atherosclerosis in CAD patients. Carotid atherosclerosis has great value in predicting coronary artery disease. The IMT can be the surrogate markers of the extensive of coronary artery lesion. 